University of Nebraska - Lincoln

DigitalCommons@University of Nebraska - Lincoln

Stephen Ducharme Publications

Research Papers in Physics and Astronomy

2005

\title{
Switching in One Monolayer of the Ferroelectric Polymer
}

Vladimir Fridkin

Institute of Crystallography, Russian Academy of Sciences, Moscow

A. levlev

Institute of Crystallography, Russian Academy of Sciences, Moscow

K. Verkhovskaya

Institute of Crystallography, Russian Academy of Sciences, Moscow

G. Vizdrik

University of Nebraska - Lincoln

S. Yudin

Institute of Crystallography, Russian Academy of Sciences, Moscow

See next page for additional authors

Follow this and additional works at: https://digitalcommons.unl.edu/physicsducharme

Part of the Physics Commons

Fridkin, Vladimir; levlev, A.; Verkhovskaya, K.; Vizdrik, G.; Yudin, S.; and Ducharme, Stephen, "Switching in One Monolayer of the Ferroelectric Polymer" (2005). Stephen Ducharme Publications. 50.

https://digitalcommons.unl.edu/physicsducharme/50

This Article is brought to you for free and open access by the Research Papers in Physics and Astronomy at DigitalCommons@University of Nebraska - Lincoln. It has been accepted for inclusion in Stephen Ducharme Publications by an authorized administrator of DigitalCommons@University of Nebraska - Lincoln. 
Authors

Vladimir Fridkin, A. levlev, K. Verkhovskaya, G. Vizdrik, S. Yudin, and Stephen Ducharme 


\title{
Switching in One Monolayer of the Ferroelectric Polymer
}

\author{
V. Fridkin, ${ }^{1,2}$ A. Ievlev, ${ }^{2}$ K. Verkhovskaya, ${ }^{2}$ G. Vizdrik, ${ }^{1}$ \\ S. Yudin, ${ }^{2}$ and S. Ducharme ${ }^{1}$
}

1 Department of Physics and Astronomy and Center for Material Research and Analysis, University of Nebraska-Lincoln, Lincoln, NE 68588-0111, USA

2 Institute of Crystallography, Russian Academy of Sciences, Moscow 119333, Russia

\begin{abstract}
The switching in one monolayer of ferroelectric vinylidene fluoride-trifluoroethylene copolymer P[VDF-TrFE] is observed. The kinetics of switching is well described by LandauKhalatnikov (LK) equation.
\end{abstract}

Keywords: Ferroelectrics, phase transitions

The investigation of the switching kinetics in the ultrathin Langmuir-Blodgett (LB) ferroelectric films of the copolymer P[VDF-TrFE] with thickness of 2-30 monolayers (ML) was performed in [1,2]. The switching kinetics of these ultrathin films is well described by the Landau-Khalatnikov equation $[3,4]$ and shows the critical behavior at $V=V_{C}\left(V\right.$ is applied voltage, $V_{C}$ is coercive voltage). For more thick films ( 100 ML) the usual exponential dependence of switching time $t_{0}$ on the applied voltage $V$ is observed, governed by the domain-nucleation mechanism of Kolmogorov-Takagi-Ishibashi.

For the first time these results were presented in 2002 on the Russian-Japanese Ferroelectric Symposium in Saint Petersburg, organized by Prof. Lemanov.

The critical behavior of the copolymer film switching may be obtained from the Landau- Khalatnikov (LK) equation for the phase transition of the first order:

$$
\xi \frac{d P}{d t}=-\frac{\partial G}{\partial P}=-a\left(T-T_{0}\right) P-\beta P^{3}-\gamma P^{5}+\frac{V}{d},
$$

where $P$ is the polarization, $G$ is the Gibbs free energy, $\xi$ is the polarization damping constant, which could be temperature dependent, $T_{0}$ is Curie temperature, $\alpha, \beta$ and $\gamma$ are the Landau-Ginzburg coefficients, $V$ is the applied voltage, and $d$ is the film thickness. The critical behavior of the switching time $t_{0}$ on $V / V_{C}$ is obtained from (1) [2]:

$$
\frac{1}{t_{0}} \cong \frac{1}{\tau}\left(\frac{V}{V_{c}}-1\right)^{1 / 2}, \quad \tau \approx 6,3 \frac{\gamma \xi}{\beta^{2}},
$$

where $V_{C}$ is coercive voltage. The Landau-Ginzburg coefficients for the copolymer are $\beta \approx-1.9 \times 10^{12} \mathrm{~J} \mathrm{~m}^{5} / \mathrm{C}^{2}, \gamma=1.9 \times 10^{14} \mathrm{~J} \mathrm{~m}^{9} / \mathrm{C}^{4}[5]$. Determination of the critical switch- 
ing time from kinetics curves for the condition $P\left(t_{0}\right)=P_{S} / 2$ gave $\xi \approx 3.6 \times 10^{10} \mathrm{~V} \cdot \mathrm{m} \cdot \mathrm{s} /$ $\mathrm{C}[2]$.

The observation of the critical switching and measurement of the $1 / t_{0}=\left(1 / t_{0}\right)(\mathrm{V} /$ $V_{C}$ ) dependence may be performed by the other method. It can be shown from the LK equation, that the initial derivative $d P / d t$ in the vicinity of $P=P_{S}$ also reveals the critical behavior:

$$
\left.\frac{d P}{d t}\right|_{P=P_{S}}=\frac{1}{\xi} E_{C}\left[\frac{V}{V_{C}}-1\right]
$$

where $E_{c}$ is the coercive field. The relation (3) is valid both for the first and second order phase transition.

Let us compare the critical switching behavior of the LK mechanism with a domain mechanism of switching, governed by the Kolmogorov-Avrami-Ishibashi (KAI) expression [6]:

$$
\begin{aligned}
2 t_{0} & =t_{0 m} \exp \left(\frac{V_{0}}{V}\right) \\
\left.\frac{d P}{d t}\right|_{P=P_{S}} & =P_{S} \frac{1}{t_{0 m}} \exp \left(-\frac{V_{0}}{V}\right)
\end{aligned}
$$

The Equations (4) and (5) show, that in KAI mechanism there is exponential dependence of switching time $t_{0}$ on $V$ and there is no peculiarities neither of $t_{0}$ nor of $d P /$ $\left.d t\right|_{P=P S}$ at the coercive voltage $V_{C}$. All the experimental data on the switching of ferroelectric crystals and films are in good agreement with KAI mechanism and (4), (5) [6]. To the contrary the experimental data for the ultrathin ferroelectric copolymer films are in good agreement with the LK mechanism and show the critical switching in accordance with (2) and (3) $[2,7]$.

It means also, that ultrathin (two-dimensional) ferroelectric films show very peculiar switching kinetics, which differs drastically from the KAI mechanism $(4,5)$.

In the present paper we investigated the switching kinetics in one ML of the ferroelectric copolymer. To do it we have prepared by the LB method [5] the combined film, consisting of two ML of ferroelectric polymer separated by two ML of the nonferroelectric anthraquinone dye (see Fig. 1). Prior measurements of the switching ki-

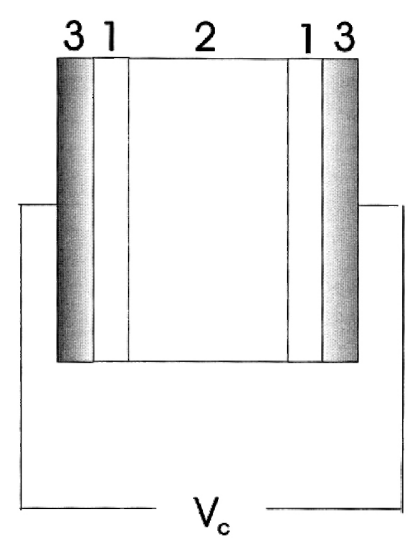

Figure 1. The scheme of the combined film: $1=$ monolayer of the ferroelectric copolymer, $d_{1}$ - thickness 5-17 $\AA, \varepsilon_{1}=7 ; 2$ = two monolayers of nonferroelectric anthraquinone dye, $d_{2}$-thickness $40 \AA, \varepsilon_{2}=3 ; 3=$ Al-electrodes. 


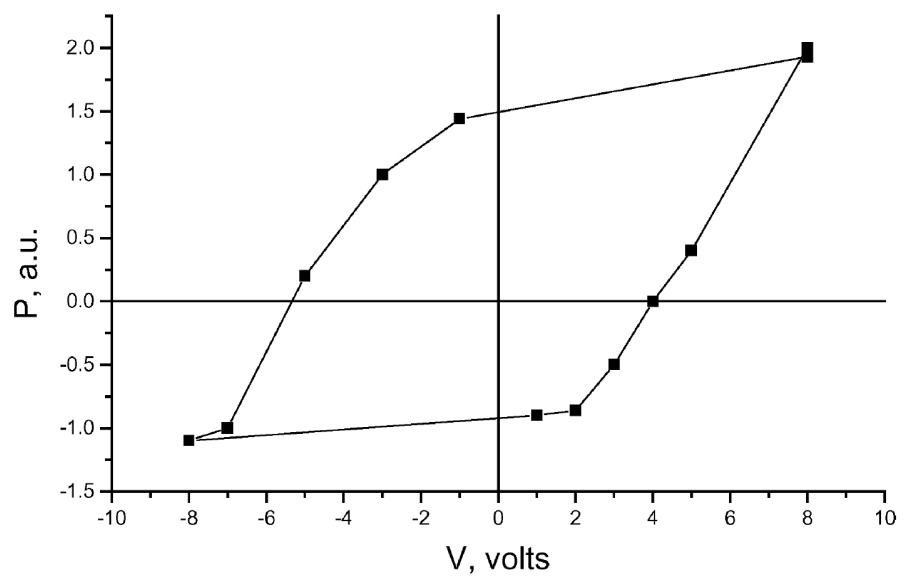

Figure 2. The hysteresis loop of the combined film.

netics the hysteresis loop and coercive voltage (4-5 volts) were obtained (Figure 2). The asymmetry of the hysteresis loop is caused by the difference in the boundary condition for each ferroelectric monolayer. The switching kinetics was measured by the Chynoweth method, described in [2].

In the present paper we have measured the initial slope of the switching kinetics curves for different $V$ (the switching took place in the direction, which corresponds $V_{C}=4$ volts). Figure 3 shows the experimental results. The critical character of the switching is evident: there is no switching at $V<V_{C^{\prime}}$ and at $V \rightarrow V_{C}, t_{0} \rightarrow \infty$. The dependence of $(d P / d t)_{P=P S}$ on $V / V_{C}$ is linear. To obtain the value of the damping parameter $\xi$ we must determine the coercive field $E_{C}$ for the combined film from Figure 2:

$$
E_{C}=\frac{V_{C}}{2 d_{1}+d_{2} \frac{\varepsilon_{1}}{\varepsilon_{2}}}
$$

where $V_{C}$ is the coercive voltage, applied to the combined film.

Substituting in (6) the dielectric constant of ferroelectric $\varepsilon_{1} \approx 7$ [5], dielectric constant of antrakhinon dye $\varepsilon_{2} \approx 3, d_{2}=40 \AA$ and $d_{1}=5-17 \AA$ (depending on LB technol-

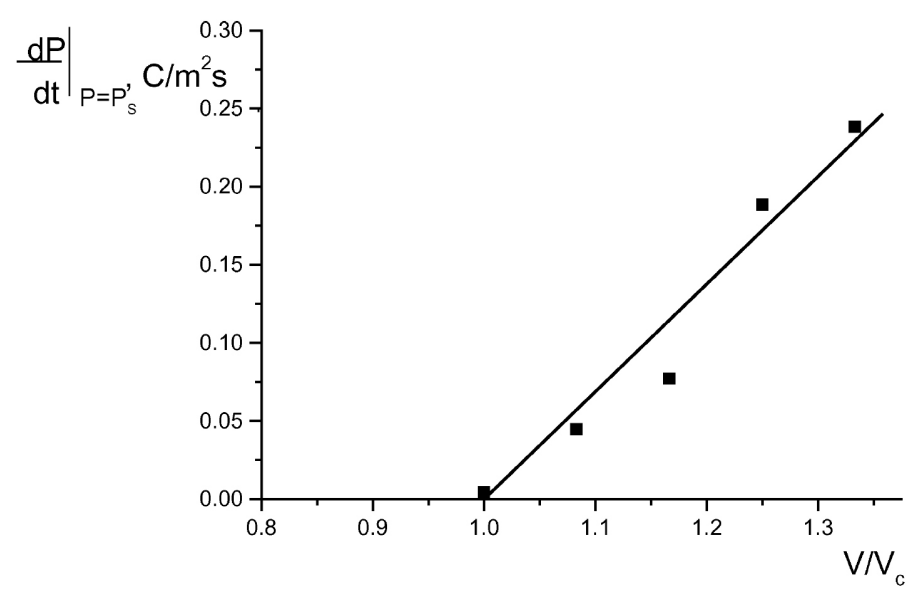

Figure 3. Dependence of $(d P / d T)_{\mathrm{P}=\mathrm{PS}}$ on $V / V_{C}$. 
ogy [8]) and neglecting internal and electrode Thomas-Fermi screening, we obtain $E_{C}$ $\approx(0.3-0.4) \times 10^{9} \mathrm{~V} / \mathrm{m}$. This value coincides very well with intrinsic Landau-Ginzburg value of coercive field in ferroelectric copolymer [1, 2].

The corresponding value of $\xi \approx(0.5-1.7) \times 10^{10} \mathrm{~V} \cdot \mathrm{m} \cdot \mathrm{s} / \mathrm{C}$ coincides with [2] by the order of value. By the present method estimation of $\xi$ does not depend on $\beta$ and $\gamma$, but supposes the realistic determination of the thickness $d_{1}$ and $d_{2}$, which depend on the LB technology [6]. Thus, the switching of combined film revealed for the first time the switching kinetics in one monolayer, which is well described by the Landau-Khalatnikov mechanism.

\section{Acknowledgments}

Work at the Institute of Crystallography of the Russian Academy of Sciences was supported by the European grant (INTAS 03-51-3967). Work at the University of NebraskaLincoln was supported by U.S. National Science Foundation and the Nebraska Research Initiative.

\section{References}

1. A. V. Bune, V. M. Fridkin, S. Ducharme, L. M. Blinov, S. P. Palto, A. V. Sorokin, S. G. Yudin, and A. Zlatkin, Nature (London), 391, 874 (1998).

2. G. Vizdrik, S. Ducharme, V. M. Fridkin, and S. G. Yudin, Phys. Rev. B 86, 094113 (2003).

3. L. D. Landau and I. T. Khalatnikov, Dokl. Akad. Nauk SSSR 96, 469 (1954).

4. V. A. Stephanovich, V. M. Fridkin, and S. Ducharme, unpublished (2004).

5. L. M. Blinov, V. M. Fridkin, S. P. Palto, A. V. Bune, P. A. Dowben, and S. Ducharme, Usp. Fiz. Nauk 170, 247 (2000).

6. Y. Ishibashi, Polarization reversal in ferroelectrics. In: C. Paz de Araujo, J. F. Scott, and G.

F. Taylor (Eds.), Ferroelectric Thin Films, volume 10, chapter 5, 135, Gordon and Breach, Amsterdam (1996).

7. L. Blinov, A. Bune, P. Dowben, S. Ducharme, V. Fridkin, S. Palto, K. Verkhovskaya, G. Vizdrik, and S. Yudin, Phase Transitions 77, 1-2, 161 (2004).

8. M. Bai, M. Poulsen, A. V. Sorokin, S. Ducharme, C. M. Herzinger, and V. M. Fridkin, J. Appl. Phys. 95, 3372 (2004). 